\section{Limited replication studies in functional magnetic resonance imaging research on taste and food}

\author{
Andy Wai Kan Yeung* \\ Oral and Maxillofacial Radiology, Applied Oral Sciences and \\ Community Dental Care, Faculty of Dentistry, \\ The University of Hong Kong, Hong Kong, China
}

\begin{abstract}
There have been many functional magnetic resonance imaging studies on taste and food. However, it is largely unknown if the findings have been replicated, or are replicable. The current survey evaluated 1568 articles on this topic, identified by Web of Science Core Collection database. Results revealed that only $0.7 \%$ of the articles were replication studies. Most of them were conceptual replications. The success rate of replication studies conducted by some of the authors coming from the original studies was 1.7 times higher than that of independent replication studies.
\end{abstract}

Keywords: Content analysis, food, highly cited articles, neuroimaging, odour, replication, taste.

WE can enjoy gourmet delicacies and our daily food because there is complex processing of taste and food information in our brain. Functional magnetic resonance imaging (fMRI) studies enable researchers to study the human brain in a non-invasive and non-harmful manner, that allow a better understanding of the neurobiology of taste and food perception, such as taste intensity ${ }^{1,2}$, hedonics $^{3}$ and quality ${ }^{4}$.

Bibliometric studies have identified highly cited neuroscience articles in general and particularly on taste and food $^{5-12}$. Meta-analyses have also attempted to pool evidence across the existing literature to find out brain locations responsible for processing taste and food information $^{13-17}$. However, it is still largely unknown if the findings resulting from taste and food fMRI studies have been replicated, and whether they are replicable. Therefore, the current study aimed to evaluate the prevalence of replication studies in the taste and food fMRI research field, and the proportion of successful replications.

The data source and search strategy mainly followed the findings of previous studies ${ }^{18-21}$. This study was based on the data provided by the Web of Science Core Collection hosted by Clarivate Analytics. The search string was: TOPIC = (flavour OR food OR odour OR taste OR smell OR olfactory OR olfaction OR tastant OR odorant OR gustatory OR gustation OR sweet OR sweetness OR salty OR saltiness OR sour OR sourness OR bitter OR bitterness OT umami OR savory OR savoury) AND $\mathrm{TOPIC}=(\mathrm{fMRI}$ OR 'functional magnetic resonance

\footnotetext{
*e-mail: ndyeung@hku.hk
}

imaging' OR 'functional MRI'). Only articles published in English were considered. The search yielded 1568 articles. After adding an additional search string of 'AND TOPIC $=\left(\text { replicat }{ }^{*}\right)^{\prime}, 25$ articles were identified and subsequently analysed.

As a conceptual replication of previous surveys done in the fields of psychology, education science, special education, and criminology ${ }^{18-21}$, the assessment of the identified articles followed their protocol. Briefly, the 25 articles were assessed with the following workflow: (1) Determine if the article collected new data with an intention to replicate a previous finding. (1a) If yes, determine the replication type (direct conceptual mixed), and go to level (2). (1b) If no, identify the reason for using the word 'replication' or its derivative (e.g. stating that replications are needed in future studies; self-replication by a subset or different sample within the same paper; concerning DNA replication; using the word 'replicating' to discuss consistency with prior findings without an intention to conduct a replication), and complete the assessment. (2) Determine the replication outcome (success, failure, or mixed), and go to level (3). (3) Identify the original study; publication year interval between the replication and the (last) original study; and if there were any authors overlapping between the original and replication studies, and go to level (4). (4) Record the citations received by the replication and original studies.

There were eleven true replication studies, which was equivalent to $0.7 \%$ of the total $(11 / 1568)$. These studies were published since 2007 and mostly during 2010s (Figure 1). Among these eleven studies, seven were direct replications and four were conceptual replications; eight were success replications and three were failures. Meanwhile, eight studies tried to replicate a single original study, one tried to replicate two studies, and two tried to replicate three studies (Table 1). The interval between the replication and the last original study ranged from 1 to 11 years (mean $\pm \mathrm{SD}, 5.4 \pm 3.7$ years). On average, the

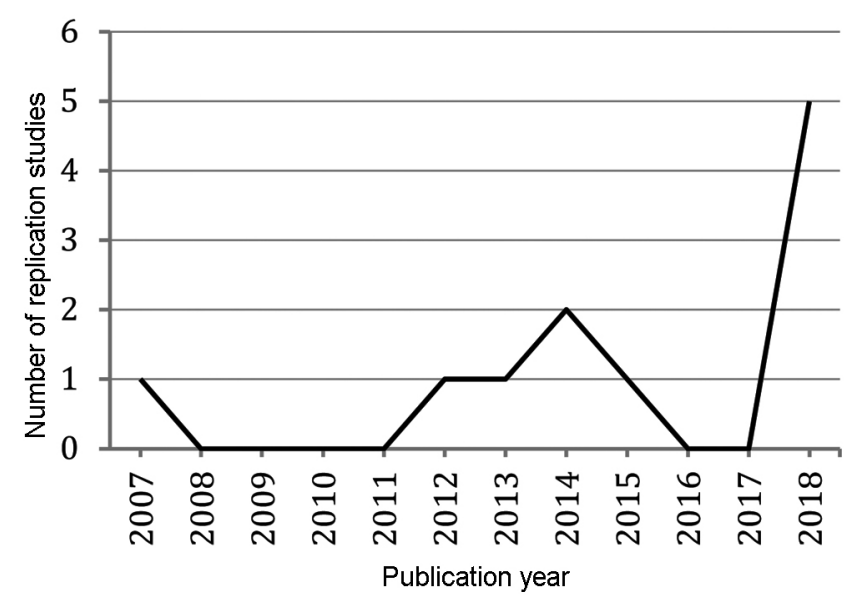

Figure 1. Distribution of publication years of replication studies. 
Table 1. Replication studies in taste and food fMRI research

\begin{tabular}{lcccc}
\hline & \multicolumn{3}{c}{ Outcome } & \\
\cline { 2 - 3 } & Success & Failure & Mixed & Total \\
\hline Type & & & & \\
$\quad$ Direct & $4(100 \%)$ & 0 & 0 & 4 \\
Conceptual & $4(57.1 \%)$ & $3(42.9 \%)$ & 0 & 7 \\
Mixed & 0 & 0 & 0 & 0 \\
Author & & & & \\
Overlap & $6(85.7 \%)$ & $1(14.3 \%)$ & 0 & 7 \\
No overlap & $2(50.0 \%)$ & $2(50.0 \%)$ & 0 & 4 \\
\hline
\end{tabular}

original studies received ten times the citations of the replication studies. The original studies had 2-474 citations (mean $\pm \mathrm{SD}, 197.8 \pm 170.9$ ), whereas the replication studies had 0-90 citations (mean \pm SD, $19.0 \pm 28.2$ ).

The remaining fourteen studies used the word 'replication' or its derivatives mainly for stating that replications were needed in future studies (as expressed by seven papers); while some of them were self-replication by a subset or different sample within the same paper (four studies); concerning DNA replication (one study); and using the word 'replicating' to discuss consistency with prior findings without an intention to conduct a replication (two studies).

The present survey has assessed 1568 taste and food fMRI articles, and revealed that $0.7 \%$ of all articles explicitly stated their attempts to replicate previously reported results. This replication rate is 5.38 times higher than that of the general education field ${ }^{18}, 1.6$ times higher than that of criminology ${ }^{21}, 1.4$ times higher than that of special education ${ }^{19}$, but slightly lower than that of the psychology field $(1.07 \%)^{20}$.

Moreover, the success rate of direct replications was 1.8 times higher than that of conceptual replications. The success rate of replication studies conducted by some of the authors coming from original studies was 1.7 times higher than that of independent replication studies. These findings also echoed with prior works regarding replications in other fields ${ }^{18-21}$, and therefore advocated the need for more independent and conceptual replication studies to ensure that successfully replicated results could be more generalizable.

Indeed, the neuroscience field has been advocating open science policy by adopting journal editorial practices that largely encourage replication studies, like the acceptance of well-designed pre-registered reports which can be replications ${ }^{22,23}$. However, as of 2017 , only few neuroscience journals $(6.0 \%)$ have explicitly welcomed replication studies in their aims and $\operatorname{scope}^{24}$. In addition, the present survey has revealed that the original studies have received citations 10 times of the replication studies, which might be a disincentive for researchers to conduct and publish replications. As fMRI is an expensive data collection modality that is more accessible by well resourced researchers ${ }^{5,6}$, it seems more likely for researchers to use it to find novel findings rather than replicating previous studies merely to confirm or rebut existing results. Similarly, due to its expensive nature, researchers tend to recruit small samples for fMRI studies ${ }^{24}$, which make replication studies more important in validating the findings. Despite all the attention we put on brain imaging studies, there is still a considerable level of subjectivity regarding the interpretation of results ${ }^{17}$. Again, this implies the development of more systematic (and systemic) approaches to replications. The research community should take extra initiatives to promote the conductance and reporting of replication studies, so that the expensive findings can be more substantiated.

We would also like to add here that there are alternative ways to identify the replication studies. For instance, a study by Cook et al. ${ }^{25}$ examined the full-text of studies with two steps: (1) Was there a statement of replication in the introduction or methods sections? and (2) Were findings from previous studies mentioned and compared to the current findings in the results and discussion sections? If answers to both questions were 'yes', then that study was defined as a replication study.

Competing interests. The author declares no competing interests.

1. Yeung, A. W. K., Tanabe, H. C., Suen, J. L. K. and Goto, T. K., Taste intensity modulates effective connectivity from the insular cortex to the thalamus in humans. Neuroimage, 2016, 135, 214222.

2. Small, D. M. and Apkarian, A. V., Increased taste intensity perception exhibited by patients with chronic back pain. Pain, 2006, 120, 124-130.

3. Small, D. M., Gregory, M. D., Mak, Y. E., Gitelman, D., Mesulam, M. M. and Parrish, T., Dissociation of neural representation of intensity and affective valuation in human gustation. Neuron, 2003, 39, 701-711.

4. Goto, T. K., Yeung, A. W. K., Tanabe, H. C., Ito, Y., Jung, H.-S. and Ninomiya, Y., Enhancement of combined umami and salty taste by glutathione in the human tongue and brain. Chem. Senses, 2016, 41, 623-630.

5. Yeung, A. W. K., Goto, T. K. and Leung, W. K., A bibliometric review of research trends in neuroimaging. Curr. Sci., 2017, 112, 725-734.

6. Yeung, A. W. K., Goto, T. K. and Leung, W. K., The changing landscape of neuroscience research, 2006-2015: a bibliometric study. Front. Neurosci., 2017, 11, 120.

7. Yeung, A. W. K., Goto, T. K. and Leung, W. K., At the leading front of neuroscience: a bibliometric study of the 100 most-cited articles. Front. Hum. Neurosci., 2017, 11, 363.

8. Kim, H. J., Yoon, D. Y., Kim, E. S., Lee, K., Bae, J. S. and Lee, J.-H., The 100 most-cited articles in neuroimaging: a bibliometric analysis. Neuroimage, 2016, 139, 149-156.

9. Yeung, A. W. K., The 100 most cited papers concerning the insular cortex of the brain: a bibliometric analysis. Front. Hum. Neurosci., 2018, 12, 337.

10. Yeung, A. W. K. and Ho, Y.-S., Identification and analysis of classic articles and sleeping beauties in neurosciences. Curr. Sci., 2018, 114, 2039-2044. 
11. Yeung, A. W. K., Identification of seminal works that built the foundation for functional magnetic resonance imaging studies of taste and food. Curr. Sci., 2017, 113, 1225-1227.

12. Kim, E. S., Yoon, D. Y., Kim, H. J., Jeon, H. J., Lee, J. Y., Cho, B.-M. and Lee, K., Citation classics in neurointerventional research: a bibliometric analysis of the 100 most cited articles. J. Neurointerv. Surg., 2017, 9, 508-511.

13. Yeung, A. W. K., Sex differences in brain responses to food stimuli: a meta-analysis on neuroimaging studies. Obes. Rev., 2018, 19, 1110-1115.

14. van der Laan, L. N., De Ridder, D. T., Viergever, M. A. and Smeets, P. A., The first taste is always with the eyes: a metaanalysis on the neural correlates of processing visual food cues. Neuroimage, 2011, 55, 296-303.

15. Yeung, A. W. K., Goto, T. K. and Leung, W. K., Basic taste processing recruits bilateral anteroventral and middle dorsal insulae: an activation likelihood estimation meta-analysis of fMRI studies. Brain Behav., 2017, 7, e00655.

16. van Meer, F., van der Laan, L. N., Adan, R. A., Viergever, M. A. and Smeets, P. A., What you see is what you eat: an ALE metaanalysis of the neural correlates of food viewing in children and adolescents. Neuroimage, 2015, 104, 35-43.

17. Yeung, A. W. K., Goto, T. K. and Leung, W. K., Affective value, intensity and quality of liquid tastants/food discernment in the human brain: an activation likelihood estimation meta-analysis. Neuroimage, 2018, 169, 189-199.

18. Makel, M. C. and Plucker, J. A., Facts are more important than novelty: replication in the education sciences. Educ. Res., 2014, 43, 304-316.

19. Makel, M. C., Plucker, J. A., Freeman, J., Lombardi, A., Simonsen, B. and Coyne, M., Replication of special education research: Necessary but far too rare. Rem. Spec. Educ., 2016, 37, 205-212.

20. Makel, M. C., Plucker, J. A. and Hegarty, B., Replications in psychology research: how often do they really occur? Perspect. Psychol. Sci., 2012, 7, 537-542.

21. Pridemore, W. A., Makel, M. C. and Plucker, J. A., Replication in criminology and the social sciences. Annu. Rev. Criminol., 2018, 1, 19-38.

22. Chambers, C. D., Registered reports: a new publishing initiative at Cortex. Cortex, 2013, 49, 609-610.

23. Poldrack, R. A. et al., Scanning the horizon: towards transparent and reproducible neuroimaging research. Nat. Rev. Neurosci., 2017, 18, 115-126.

24. Yeung, A. W. K., Do neuroscience journals accept replications? a survey of literature. Front. Hum. Neurosci., 2017, 11, 468.

25. Cook, B. G., Collins, L. W., Cook, S. C. and Cook, L., A replication by any other name: a systematic review of replicative intervention studies. Rem. Spec. Educ., 2016, 37, 223-234.

Received 31 October 2018; revised accepted 30 May 2019

doi: $10.18520 / \mathrm{cs} / \mathrm{v} 117 / \mathrm{i} 8 / 1345-1347$

\section{Use of a weather forecast model to identify suitable sites for new wind power plants in Karnataka}

\author{
Anasuya Gangopadhyay ${ }^{1, *}$, Radhika Kanase ${ }^{2}$, \\ Greeshma Mohan ${ }^{2}$, Madhuparna Halder ${ }^{2}$, \\ Medha Deshpande ${ }^{2}$, Parthasharathi \\ Mukhopadhyay $^{2}$ and J. Srinivasan ${ }^{1}$
}

${ }^{1}$ Divecha Centre for Climate Change, Indian Institute of Science, Bengaluru 560 012, India

${ }^{2}$ Indian Institute of Tropical Meteorology, Dr Homi Bhabha Road, Pune 411 008, India

The wind speed forecast by a weather forecast model (with a resolution of $3 \mathrm{~km}$ ) has been used to identify regions with high wind speed in Karnataka. The correlation between daily wind speed predicted by the model and the measured wind speed at three stations in Karnataka has been shown to be high. Based on this model, new locations have been suggested for the installation of new wind power plants in Karnataka.

Keywords: Karnataka, potential site, renewables, weather model, wind plant.

THE installed capacity of wind power plants in India is expected to reach 60 Gigawatts (GW) by 2022 . Karnataka is expected to attain an installed capacity of $6.2 \mathrm{GW}$ (ref. 1). At the end of June 2018, the total installed wind capacity of Karnataka was $4.7 \mathrm{GW}$ (ref. 2). In order to identify the regions suitable for the location of the new wind power plants, grid stability and environmental impact need to be considered. The majority of the wind power plants in Karnataka are owned by independent power producers (IPPs). The locations of wind power plants where the average wind speed is higher compared to other places would mean more energy generation. Hence, locating high wind speed regions of the state is important. Some of the areas with high-wind in Karnataka have many wind power plants and hence may not be suitable for new power plants. So, it is a critical task to identify areas where the wind speed is reasonably high and there is a possibility of installation of new wind power plants. Wind speed varies dramatically over small distances due to local orography. We do not have sufficient number of stations with wind data to identify the areas suitable for new wind power plants. Wind is intermittent by nature and fluctuates in time scales from seconds up to a season. The increasing share of wind energy in the energy mix of the state will pose a new challenge for grid stability. The variability of wind energy decreases when energy is fed to the grid from geographically distributed wind power plants. This is called geographic smoothing ${ }^{3}$. In Karnataka,

*For correspondence. (e-mail: anasuyagangopadhyay@gmail.com) 